\title{
Neue Werkstoffe für textile Sensoren zur Körperfunktionsüberwachung
}

\author{
T. Quadflieg ${ }^{1}$, T. Schlebusch ${ }^{2}$, M. Ulbrich ${ }^{2}$, S. Jockenhövel ${ }^{1}$, S. Leonhardt ${ }^{2}$ und T. Gries ${ }^{1}$ \\ ${ }^{1}$ Institut für der Textiltechnik; RWTH Aachen University; Aachen; Deutschland \\ ${ }^{2}$ Philips Lehrstuhl für Medizinische Informationstechnik an der RWTH Aachen University; Aachen; \\ Deutschland
}

\section{Zusammenfassung}

In diesem Beitrag werden signalverbessernde Beschichtungsverfahren für textile Sensoren zur Körperfunktionsüberwachung vorgestellt. Dabei werden auf ein Silberfaden Poleyruthandirspersionen in Dünnstbeschichtungen aufgetragen und über Normwaschversuche einer chemischen und mechanischen Belastung unterzogen. Die Widerstandsfähigkeit von elektrisch leitenden Garnen gegenüber diesen Balsastungen lässt darüber steigern. Verbessert wird die strukturelle Stabilität (textile Struktur) und die Oberflächenleitfähigkeit.

\section{Einleitung}

Die telemedizinische Überwachung von Vitalparametern birgt ein bislang noch nicht ausgeschöpftes Potential zur Verbesserung der medizinischen Versorgung und zu nachhaltigen Kosteneinsparungen im Gesundheitswesen. Der vielversprechendste Bereich für Telecare und -monitoring ist der Heimpflegebereich. Stationäre Aufenthalte von Risikopatienten können durch mobile Überwachung beachtlich verkürzt werden. Dies bedeutet eine Kostensenkung bei gleichzeitiger Steigerung der Lebensqualität.

Durch in Bekleidung integrierte Sensorik können Personen mit Gesundheitsproblemen dauerhaft und komfortabel medizinisch überwacht werden. Eine Integration von textiler Sensorik (Elektroden, Leiterbahnen) in die Bekleidung reduziert das Gewicht und erhöht den Tragekomfort gegenüber konventionellen Klebeelektroden. [1]

Textile Elektroden werden aus leitfähigen Garnen in textilen Prozessen (stricken, wirken) zu einer Fläche verarbeitet und anschließend konfektioniert. Bisherige leitfähige Garne haben den Nachteil einer unzureichenden mechanischen Stabilität der leitfähigen Schicht. Insbesondere durch Waschen wird die leitfähige Schicht beschädigt und die Signalqualität verschlechtert. [2][3]

\section{Forschungsergebnisse}

Untersucht werden neuartige polymerbasierte Beschichtungen für den Einsatz körpernah getragener Sensorik. Als Ausgangsmaterial dient silberbeschichtetes Polyamid, welches durch Dünnstbeschichtung die Stabilität der textilen Elektroden verbessert. Dabei werden die folgenden drei Ansätze untersucht:

- Beschichtung der Garne

- Beschichtung der textilen Fläche

- Funktionalisierung der Beschichtung mit Carbon Nano Tubes (CNTs)

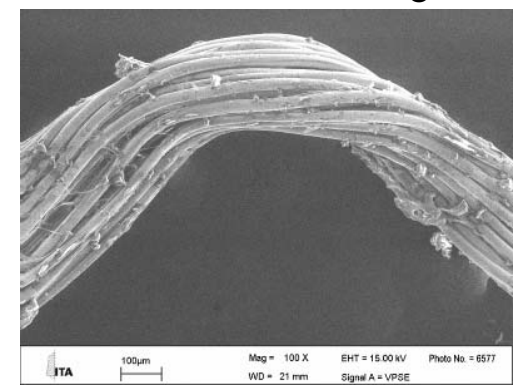

Bild 2 beschichtetes Garn

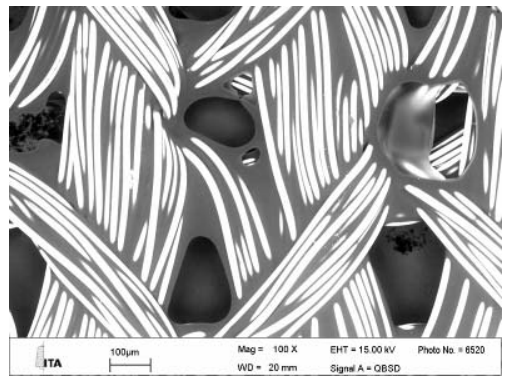

Bild 3 beschichtete Fläche

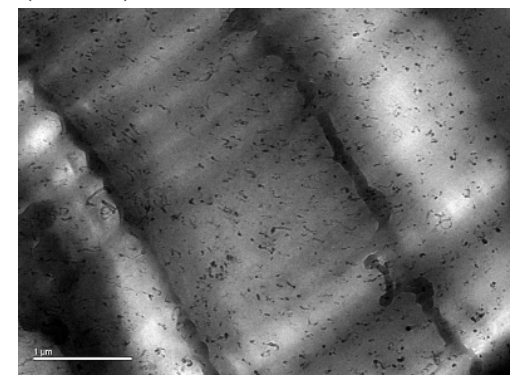

Bild 4 Beschichtung mit CNTs 
Der Einsatz der so gefertigten textilen Elektroden elektrotechnisch durch den Übergangswiderstand zur Haut (Oberflächenleitfähigkeit) charakterisiert. Die Elektroden werden nach DIN EN ISO 6330 gewaschen und anschließend auf Funktionalität geprüft. Es zeigt sich, dass sich die mechanische Stabilität und damit die Signalqualität der textilen Sensoren mit polymeren Beschichtungen signifikant steigern lässt. [4]

\subsection{Beschichtungsauftrag}

Aufgrund ihrer besonders hohen elektrischen Leitfähigkeit werden für alle Versuche zweifach gezwirnte Silbergarne mit 235 dtex verwendet. Die Silbergarne bestehen aus einem PA 6.6 (Nylon)-Garn, dass nach dem Spinnen mit 99\%-igem reinem Silber beschichtet wird. Da die Oberfläche von Polyamid (PA) nicht glatt ist kann das Silber optimal darauf haften. Eine Besonderheit der Silbergarne sind seine antibakteriellen und antistatischen Eigenschaften, sowie die hohe thermische Leitfähigkeit. Zudem lassen sich die Silbergarne wie unbeschichtete synthetische Garne weiterverarbeiten. [5][6]

Als Beschichtungsmasse werden Polyurethan-Elastomere (TPU), also thermoplastische Elastomere (TPE), verwendet. Die TPE verbinden die gummielastischen Eigenschaften von Elastomeren mit dem Vorteil der Verarbeitbarkeit von Thermoplasten, wie z.B. Rezyklierbarkeit, Schweißbarkeit und Transparenz. Bei TPU handelt es sich um ein Block-Copolymer, das durch die Polyaddition von aliphatischen Diisocyanaten mit langkettigen Diolen entsteht. Das TPU wurde als wässrige Dispersion mit einem Feststoffgehalt von 23\% und 29\% verarbeitet. Zum Starten der Vernetzungsreaktion wird der Dispersion sieben Gewichtsprozent hydrophiles, aliphatisches Polyisocyanat auf Basis von Hexamethylendiisocyanat (HDI) als Härter zugeführt. Um die notwendige Verbindung der Filamente im Inneren herzustellen wird das Substrat nach der oberflächigen Beschichtung zwischen zwei Quetschwalzen hindurch geführt, die mit einem einstellbaren Druck gegeneinander pressen. Beim Abquetschen der Proben wird die Luft zwischen den Filamenten und überschüssige Beschichtungsmasse aus dem Material gedrückt. Verlässt das Band das Quetschwerk wieder, führt der Schwammeffekt des entstehenden Unterdrucks in den Zwischenräumen dazu, dass Beschichtungsmasse hineingezogen wird. So werden die Garnfilamente auch im Inneren des Garns benetzt und vor mechanischer Beanspruchung geschützt.

Bei der Benetzung des Substrats wurde insbesondere eine wesentlich flächigere, homogenere Verteilung der Beschichtungsmasse durch eine 29\%, anionische, aromatische Polyetherpolyurethan-Dispersion erreicht. Dabei handelt es sich um 4,4'-Dipenylmethendiisocyanat (MDI) angereichertes Ausgangsmaterial. Dieser aromatische Härter besitzt zwei ringförmige Aromatenstrukturen, in denen die Elektronen delokalisiert als $\pi$-Elektronenorbital vorliegen. Aus diesem Grund sind Aromaten energieärmer und deshalb weniger reaktiv im Vergleich zu nicht-aromatischen Systemen wie dem HDI. Insbesondere neigen sie nicht zu Additionsreaktionen. Im Gegensatz $\mathrm{zu}$ Beschichtungen mit HDI verringern sich mit MDI die zwischenmolekularen Anziehungskräfte, wodurch die Oberflächenspannung abnimmt und damit die Benetzungsfähigkeit zunimmt. Dies hat auch eine gleichmäßigere Verteilung der CNT zur Folge.

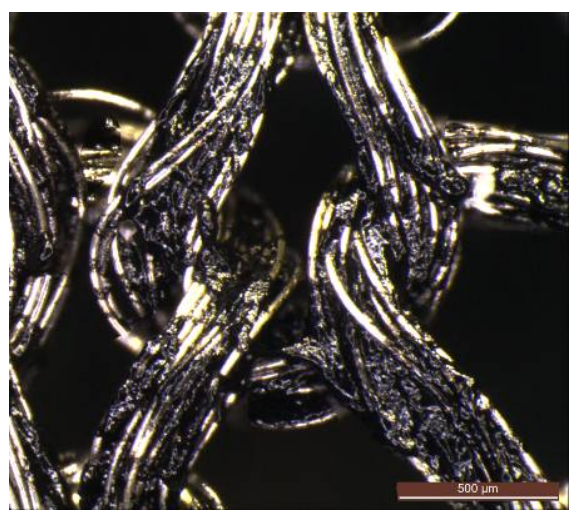

Bild 7 HDI beschichtetes Silber

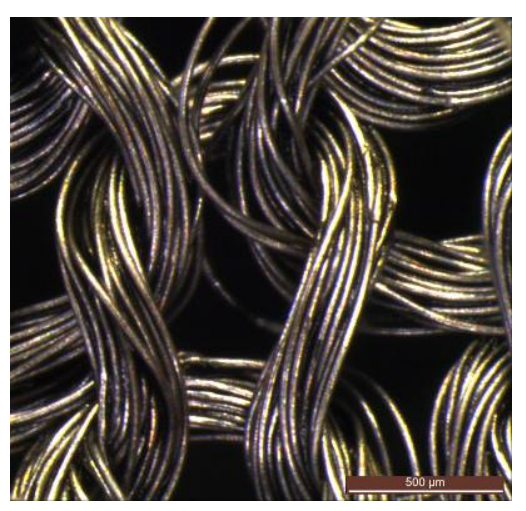

Bild 8 MDI beschichtetes Silber

Beim beschichten von Silbersubstraten mit MDI zeigt sich eine homogene Verteilung der Beschichtung mit Schichtdicken von wenigen Nanometern. 


\subsection{Waschuntersuchungen}

Beschichtung bewirkt eine mechanische Makro- und Mikrostabilisierung, d.h. die textile Struktur bleibt über die Wäschen länger erhalten:

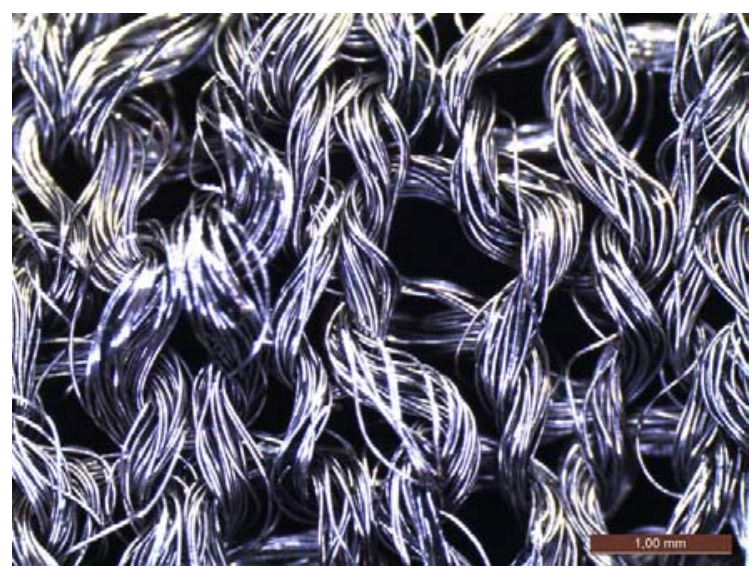

Bild 9 MDI beschichtetes Silber nach 20 Wäschen

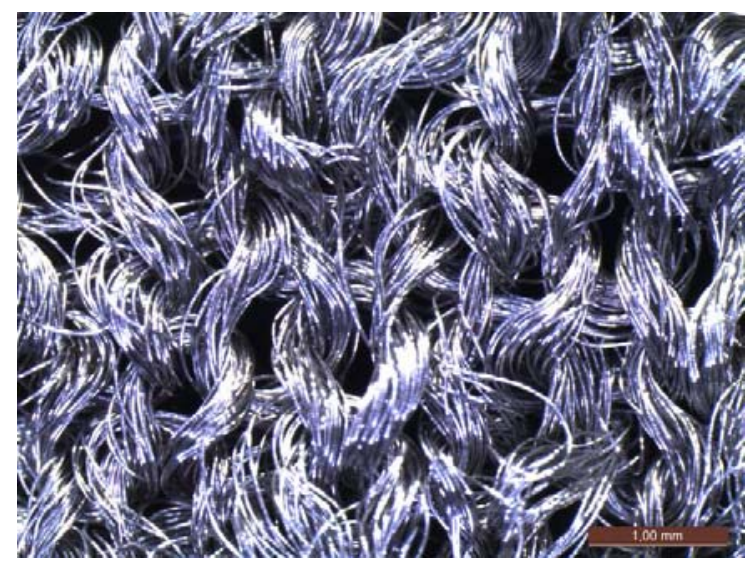

Bild 9 unbeschichtetes Silber nach 20 Wäschen

Nach 20 Waschzyklen sind die beschichteten Silbergestricke weniger beschädigt, als die unbeschichteten. Die Filamente liegen näher beieinander und die Schlaufen der Strickstruktur sind gleichförmiger. Dieser Effekt lässt sich über die Waschzyklen linear reproduzieren.

\subsection{Elektrotechnische Validierung}

Zur objektiven Bewertung der Eigenschaften von Materialien für den Einsatz als textile Elektrode können keine Messungen an Probanden herangezogen werden, da hier die Messergebnisse von einer Vielzahl von Faktoren beeinflusst werden und so die Reproduzierbarkeit solcher Messungen nicht gegeben ist. Um dies zu gewährleisten, wurde ein Elektrodenprüfstand entwickelt, welcher die komplexe Impedanz von Elektrode, Elektrodenübergang und Gewebe unter der Variation gewisser Randbedingungen messen kann (Bild 9). Die komplexe Impedanz wurde mittels eines Impedanzmessgerätes (Agilent E4980a LCR meter) gemessen.

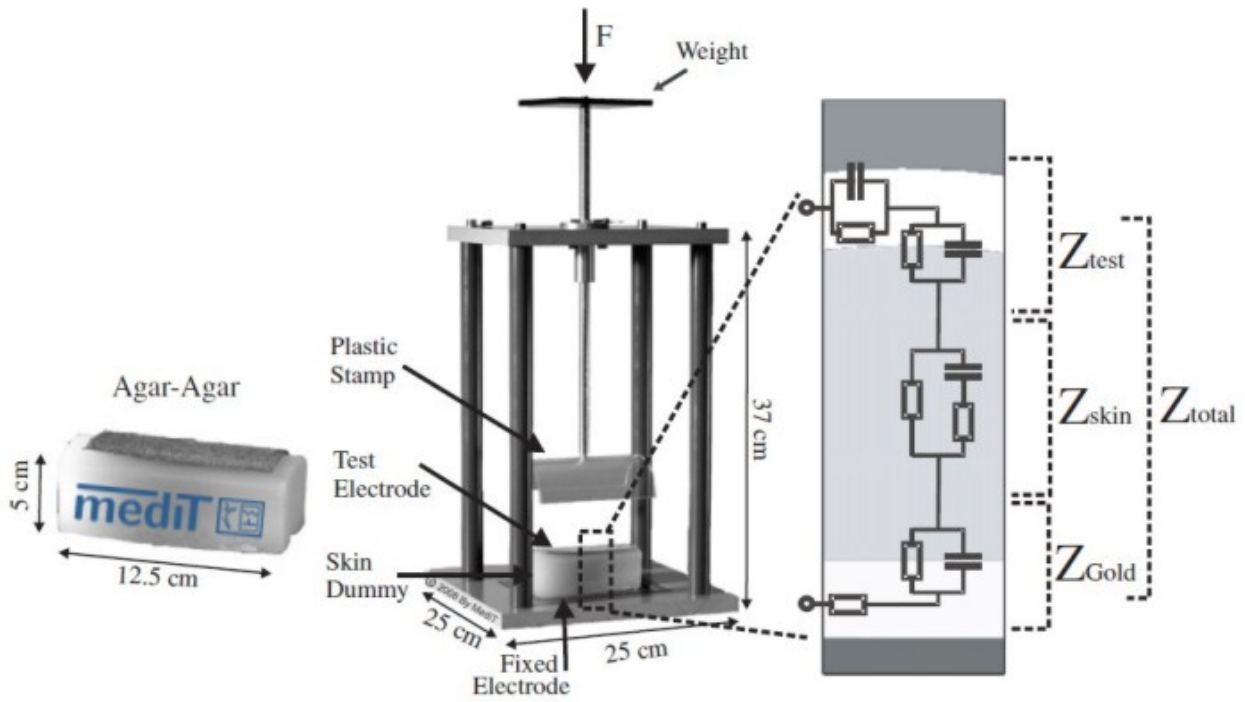

Bild 9 Bioimpedanzprüfstand

Der Teststand kann in drei Teile unterteilt werden: Das zu charakterisierende Material, einen Haut-Dummy und eine zweite Elektrode unterhalb des Dummys, welche mit Gold beschichtet wurde. Über eine solche Konfiguration, kann die komplexe Impedanz $\left(\mathrm{Z}_{\text {total }}\right)$ über eine 2-Punkt-Messung bestimmt werden. Der Dummy wurde mit Agar-Agar, einem Geliermittel welches aus Algenzellen gewonnen wird, hergestellt. Dabei wurde die Leitfähigkeit des Dummys mit Salz auf $29.3 \mu \mathrm{S} \mathrm{cm}-1$ eingestellt, welches der Leitfähigkeit nasser Haut bei $10 \mathrm{kHz}$ entspricht [7]. Um eine hautähnliche Konsistenz zu erhalten, wurden ein Mischungsverhältnis von 7g Agar-Agar zu 100 ml Wasser verwendet. Zur Messung wird die Testelektrode 
auf der Oberfläche des Dummys mittels eines Stempels fixiert. Durch das Variieren von Gewichten auf dem oberen Teil des Stempels können verschiedene Kontaktkräfte eingestellt werden, um die Abhängigkeit der Übergangsimpedanz von verschiedenen Drücken zu analysieren.

Durch Messungen an Probanden wurden Kontaktdrücke zwischen $0 \mathrm{~N}$ und $10 \mathrm{~N}$ erfasst, welche sowohl stabile Messungen erlauben als auch den Tragekomfort des Textils nicht einschränken. Deshalb wurden die Übergangsimpedanzen für diesen Bereich untersucht.

Die komplexe Impedanz wurde in einem Frequenzbereich von $5 \mathrm{kHz}$ bis $1 \mathrm{MHz}$ bestimmt, welches dem Standardbereich für Bioimpedanz-Spektroskopie-Messungen entspricht. Als Messergebnis erhält man die komplexe Gesamtimpedanz $\left(Z_{\text {total }}\right)$ welche sich aus der Impedanz der Testelektrode $\left(Z_{\text {test }}\right)$, des Haut-Dummys $\left(Z_{\text {skin }}\right)$ und der Goldelektrode $\left(Z_{\text {gold }}\right)$ zusammensetzt. Da $Z_{\text {skin }}$ und $Z_{\text {gold }}$ für alle Messungen eines Textils konstant bleiben, beruht die Impedanzänderung auf der Änderung von $Z_{\text {test, }}$ d.h. auf der Impedanz der textilen Elektrode und der Kontaktimpedanz. Alle Messungen wurden für jedes Textil und jeden Druck zehnmal wiederholt um die Güte und die Reproduzierbarkeit des Testaufbaus zu gewährleisten. Bild 10 zeigt die Druckabhängigkeit der komplexen Gesamtimpedanz eines Textils, sowie die Erhöhung der Impedanz nach 20 Waschzyklen.
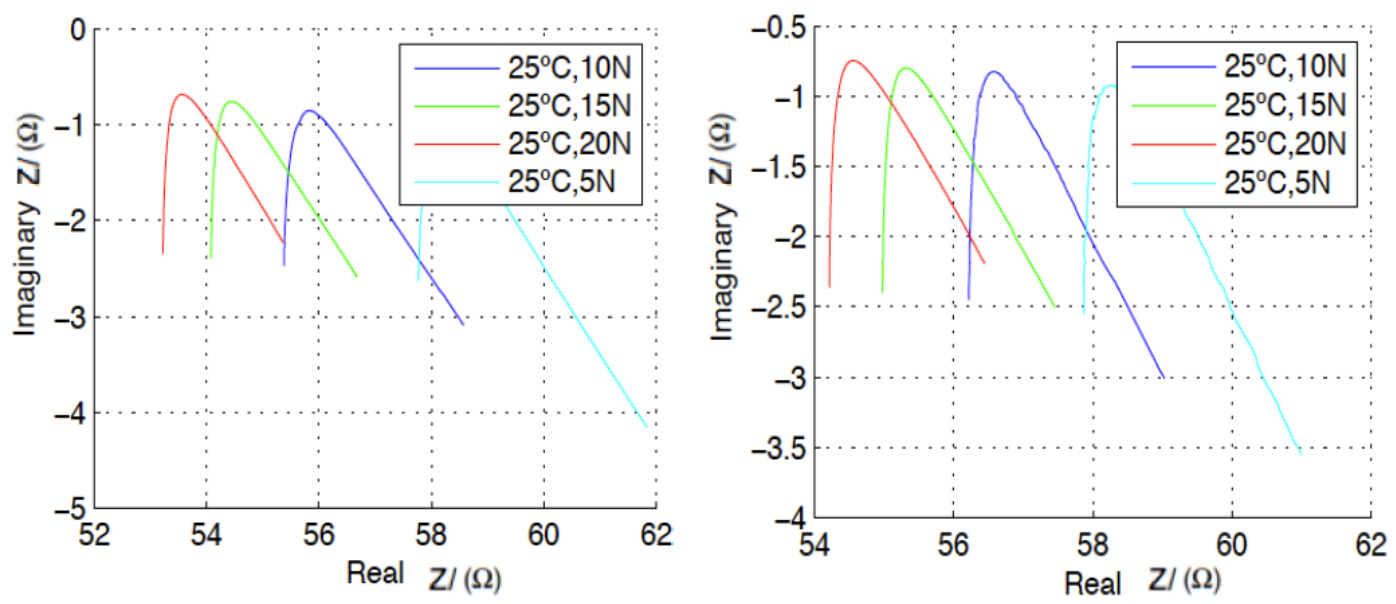

Bild 10 Impedanzmessungen eines Textils ohne Wäsche (links), nach 20 Waschzyklen (rechts)

\section{Fazit}

Die mit Polyurethandispersionen beschichteten Silbergestricke weisen eine bessere mechanische Stabilität gegenüber Belastungen auf. Nach 20 Waschzyklen degradiert unbeschichtetes Silbergestrick deutlich in seiner Struktur und in seiner Funktion. Durch Dünnstbeschichtungen die vorrangig zwischen den Filamenten liegen bleibt die Oberflächenleitfähigkeit von Elektroden aus Silbergestrick nahezu erhalten, während ihre Waschbarkeit verbessert wird.

\section{Literatur}

[1] L. Beckmann, C. Neuhaus, G. Medrano, N. Jungbecker, M. Walter, T. Gries, S. Leonhardt, Physiological Measurement, Vol. 31, 2010, pp. 233-247

[2] Locher, I.; Kirstein, T.; Tröster, G.: "From Smart Textiles to Wearable Systems", mst news (2005), Heft 2/05, Seite 12-13, 2005

[3] M. Catrysse, R. Puers, C. Hertleer, L. Van Langenhove, H. Van Egmond, D. Matthys, Sensors and Actuators A - Physical 114 (2004) 302.

[4] DIN EN WAschen

[5] D. Hegemann, M. Amberg, A. Ritter and M. Heuberger, Materials Technology, 2009, Vol. 24(1), pp.4145

[6] J. Scholz, G. Nocke, F. Hollstein and A. Weissbach, Surface \& Coatings Technology 192 (2005) 252256

[7] C. Gabriel, S. Gabriel, E. Corthout: The dielectric properties of biological tissues, Physics in Medicine and Biology, Volume 41, Number 11, pp. 2231-2249 